\section{Aprepitant auch bei moderat emeto- gener Chemotherapie wirksam}

Der Neurokinin-1-Rezeptorantagonist Aprepitant $\left(\right.$ Emend $\left.^{\circledR}\right)$ ist nicht nur zur Prophylaxe von Übelkeit und Erbrechen bei Patienten mit hoch emetogener cisplatinhaltiger Chemotherapie hocheffektiv, sondern auch bei moderat emetogener Chemotherapie wirksam und verträglich. Unter der Kombination mit Aprepitant konnte das Erbrechen bei $76 \%$ vollständig verhindert werden, während dies nur bei $59 \%$ in der Kontrollgruppe der Fall war.

Eine bei Brustkrebspatientinnen oft eingesetzte Behandlungsoption, und damit eine der häufigsten Chemotherapien überhaupt, ist die Kombination von Cyclophosphamid mit Anthrazyklin. Obwohl diese
Therapie nur als moderat emetogen gilt, führt sie bei den behandelten Frauen überdurchschnittlich häufig zu Übelkeit und Erbrechen. In die doppelblinde, multizentrische Studie wurden randomisiert 866 Frauen mit Brustkrebs einbezogen, die erstmals mit Cyclophosphamid mit/ohne Doxorubicin oder Epirubicin behandelt wurden. Gegen Übelkeit und Erbrechen erhielten sie entweder im Kontrollarm Ondansetron plus Dexamethason am 1. Tag, gefolgt von der Gabe von Ondansetron an den Tagen 2 und 3 oder Aprepitant plus Ondansetron plus Dexamethason am 1. Tag, gefolgt von der Gabe von Aprepitant an den Tagen 2 und 3. Der primäre Endpunkt, definiert als kein Erbrechen und kein Bedarf an zusätzlicher antiemetischer Medikation innerhalb von 5 Tagen nach Beginn der Chemotherapie, wurde von $51 \%$ der Patientinnen in der Aprepitantgruppe und von $43 \%$ in der Kontrollgruppe erreicht.

Quelle: 29. Kongress der European Society for Medical Oncology (ESMO), 29.10.2.11.2004, Wien. J. Herrstedt: Randomized, double-blind trial comparing the effect of an aprepitant regimen versus an antiemetic standard regimen during four cycles of moderately emetogenic chemotherapy. Session Supportive Care am 31.10.04 (abstr 827).

Weitere Informationen bei MSD SHARP \& DOHME GMBH

Lindenplatz 1, 85540 Haar

Tel. +498945612612

E-Mail infocenter@msd.de

\section{"Targeted Therapy" mit}

\section{Tyrosinkinase-Hemmer Erlotinib: Signifikante Überlebensverlän- gerung bei Patienten mit fortge- schrittenem NSCLC}

Trotz neuer Chemotherapien war die Prognose von Patienten mit fortgeschrittenem nicht-kleinzelligen Lungenkarzinom (NSCLC) bisher immer noch ungünstig. Ein entscheidender Durchbruch konnte nun erstmals mit Erlotinib (Tarceva ${ }^{\circledR}$ ) erzielt werden, das als erster Vertreter der neuen Wirkstoffklasse der oralen Tyrosinkinase-Inhibitoren bei mehrfach vorbehandelten Patienten mit NSCLC das Überleben signifikant verlängert.
Die Wirksamkeit des Tyrosinkinase-Hemmers konnte in einer internationalen, prospektiven, randomisierten und Plazebokontrollierten Phase-III-Studie (BR.21) bewiesen werden (Shepherd F. et al.). Die Studie umfasste insgesamt 731 Patienten mit NSCLC im Stadium III b oder IV, die zuvor mit ein bis zwei Chemotherapien vorbehandelt worden waren.

Unter Therapie mit dem TyrosinkinaseHemmer betrug die mediane Überlebenszeit 6,7 Monate im Vergleich zu 4,7 Monaten nach Gabe von Plazebo $(\mathrm{p}=0,001)$, was einer Verbesserung von $43 \%$ entspricht. Nach einem Jahr lebten noch $31 \%$ der Patienten unter Erlotinib, im Plazebo-Arm nur 21\% der Probanden.
Zudem ließ sich das progressionsfreie Überleben von 8,0 auf 9,7 Wochen verlängern $(\mathrm{p}<0,0001)$. Die bewiesene Wirksamkeit von Erlotinib ist vergleichbar mit der Wirksamkeit der üblicherweise eingesetzten Chemotherapie. Ein großer Vorteil des Tyrosinkinase-Hemmers: Er ist nicht mit den typischen Nebenwirkungen von Zytostatika verbunden.

Erlotinib ist in den USA als Second- und Third-line-Therapie bereits seit November 2004 zugelassen. Die europaweite Zulassung wird für das 4. Quartal 2005 erwartet.

Weitere Informationen bei

Dr. med. H.-U. Jelitto

Hoffmann-La Roche AG

Tel. +49 7624 14-2400, Fax -3366

\title{
PharmaTicker+++ PharmaTicker+++ PharmaTicker+++ PharmaTicker+++
}

Hoffmann-La Roche AG. Das orale Flouropyrimidin Capecitabin (Xeloda ${ }^{\circledR}$ ) hat jetzt die Zulassung zur adjuvanten Therapie von Patienten nach der Operation eines Kolonkarzinoms im Stadium III (Dukes Stadium C) erhalten. Das 5-Fluorouracil-Prodrug ist eine effektivere und zugleich besser verträgliche Alternative zur intravenösen Gabe von 5-Fluorouracil/Leucoverin (5-FU/LV)

Weitere Informationen bei

Hoffmann-La Roche AG, Dr. med. H.-U. Jelitto

Tel. +49 7624 14-2400, Fax -3366
Pfizer Oncology GmbH. In der IES-Studie konnte das Rezidivrisiko durch den Switch nach 2-3 Jahren Tamoxifen auf den Anti-Aromatase-Wirkstoff Exemestan (Aromasin ${ }^{\circledR}$ ) um $27 \%$ signifikant gesenkt werden. Exemestan ist derzeit zugelassen zur Therapie des fortgeschrittenen Mammakarzinoms nach Progress unter Anti-Östrogentherapie. Die erweiterte Zulassung für die FAST (Frühe Adjuvante Sequenz-Therapie) wurde im Dezember 2004 beantragt.

Haas \& Health Partner, Annette Haas

Tel.: +49 6123 7057-20

E-mail a.haas@haas-health.de
Ferring GmbH. Eine auf dem XX. Kongress der European Association of Urologists (EAU) im März in Istanbul vorgestellten Phase-II-Eskalationsstudie zeigt vielversprechende Aussichten für die Anwendung von Degarelix von Ferring bei der Behandlung von Prostatakrebs. Aufgrund dieser ermutigenden Ergebnisse steht Degarelix derzeit vor dem Eintritt in klinische Studien der Phase III.

Weitere Informationen bei ipse Communication, Kai Weller Tel. +49 30 288846-20, Fax -46 E-Mail k.weller@ipse.de 


\section{RTOG-Studie 85-31: \\ Prostatakrebs - Verlängertes Überleben durch adjuvante Zoladex $^{\circledR}$-Therapie}

Eine mit der Radiotherapie begonnene Zoladex $^{\circledR}$ (Goserelin)-Behandlung bei Patienten mit Prostatakrebs verbessert das progressionsfreie Überleben und das $\mathrm{Ge}$ samtüberleben. Dies sind die Ergebnisse einer Langzeit-Studie, die in der Aprilausgabe des International Journal of Radiation Oncology, Biology and Physics veröffentlicht wurden. Die unabhängige, von der Radiation Therapy Oncology Group initiierte Studie (RTOG-Studie 85-31), die nahezu 1000 Patienten mit lokal fortge- schrittenem Prostatakarzinom einschloss, ist eine der größten dieser Art.

Ziel war die Überprüfung der Wirksamkeit einer mit der Radiotherapie begonnenen androgensuppressiven Therapie mit Zoladex $^{\circledR}$ bei Patienten mit ungünstiger Prognose. Eingeschlossen wurden insgesamt 977 Patienten mit lokal fortgeschrittenem Prostatakarzinom. Sie erhielten entweder eine Bestrahlung direkt gefolgt von einer monatlichen Zoladex ${ }^{\circledR}$-Gabe, die in der letzten Woche der Bestrahlung begonnen und bis zur Progression fortgesetzt wurde ( $n=477)$, oder eine alleinige Radiotherapie mit Beginn der Zoladex ${ }^{\circledR}$-Therapie nach belegter Progression $(n=468)$. Nach einer Nachbeobachtungszeit von median 7,6 Jahren zeigten sich signifikante Vorteile bei allen Endpunkten im Studienarm, der die adjuvante Gabe von Zoladex ${ }^{\circledR}$ vorschrieb.

«Für Patienten mit schlechter Prognose ist die adjuvante Gabe von Zoladex ${ }^{\circledR}$ nach Radiotherapie nicht nur mit einer Reduktion des Progressionsrisikos assoziiert, sondern auch mit einer signifikanten Verbesserung des Überlebens», so Dr. Collen Lawton von der RTOG: «Bei Patienten mit lokal fortgeschrittenem Tumor wird die Langzeitgabe von Zoladex ${ }^{\circledR}$ zusätzlich zur Radiotherapie ein wertvoller Therapiestandard werden.»

Weitere Informationen bei

Publicis Vital PR, Eva Radas

Tel. +49 221 912719-36, Fax -7

E-mail eva.radas@publicis-vital-pr.de

\section{Fujirebio Diagnostics bringt ersten Bluttest für Mesotheliome auf den Markt}

Fujirebio Diagnostics, Inc. (FDI), ein weltweit führendes Unternehmen auf dem Gebiet der onkologischen Diagnostik, und Cisbio international, ein französisches Biotechnologie-Unternehmen, gaben Anfang April den Abschluss einer Vertriebsvereinbarung für den europäischen Vertrieb von FDIs neuem Bluttest für Mesotheliome (MESOMARK $^{\mathrm{TM}}$ ) bekannt. Diese Vereinbarung, die zweite für FDI, setzt die weltweite Markteinführung von MESOMARK fort. Anfang des Jahres führte FDI MESOMARK in Australien ein, wo das Unternehmen einen Vertrag mit einem Vertriebsunternehmen mit Sitz in Australien abschloss.
Bisher gab es keine verlässlichen SerumTumormarker für Mesotheliome; dies bedeutet, dass die Diagnose, das Screening und das Monitoring der Reaktionen auf die Therapie schwierig waren. MESOMARK, ein manueller, enzymgebundener und immunabsorbierender Test (ELISA) zur Handhabung von Mesotheliomen, identifiziert eine Gruppe von molekularen Markern, genannt lösliche mesothelin-verwandte Proteine (Soluble Mesothelin-Related Proteins, SMRP). Diese Proteine werden von Mesotheliomzellen im Blutkreislauf freigesetzt. Da der SMRP-Spiegel mehrere Jahre vor der eigentlichen Diagnose eines Mesothelioms erhöht sein kann, hilft MESOMARK bei der Routineüberwachung von Personen, die in ihrem Umfeld Asbest ausgesetzt sind, da für diese Personengruppe das höchste Risiko der Entwicklung eines Mesothelioms besteht.

Mesotheliome entstehen hauptsächlich durch beruflich bedingten Kontakt mit Asbest. In den letzten fünf Jahrzehnten kamen über 100 Millionen Menschen weltweit bei ihrer Arbeit mit Asbest in Kontakt und es wird erwartet, dass jedes Jahr 10000 neue Fälle in den Industrieländern diagnostiziert werden. Neuere epidemiologische Studien prognostizieren einen Anstieg dieser Zahl in den nächsten Jahrzehnten.

Weitere Informationen bei Fuechtner Public Relations Ariane Füchtner

Tel. +49 30 62-989756, Fax -732682

E-mail info@fuechtner.com

\section{PharmaTicker+++ PharmaTicker+++ PharmaTicker+++ PharmaTicker+++}

AstraZeneca GmbH. Im Rahmen des «4th Global Breast Cancer Summit» (11.-13. März 2005 in Sitges, Spanien) diskutierten internationale Experten den State of the Art in der endokrinen Therapie des Mammakarzinoms. Ein Fazit: Der Aromatasehemmer Anastrozol (Arimi$\left.\operatorname{dex}^{\circledR}\right)$ ist die Therapie der Wahl beim frühen, hormonrezeptorpositiven Mammakarzinom bei postmenopausalen Frauen und hat den bisherigen Goldstandard Tamoxifen abgelöst.

Weitere Informationen bei

Publicis Vital PR, Natalie Fiebig

Tel. +49 221 912719-85, Fax -47

E-mail natalie.fiebig@publicis-vital-pr.de
Mundipharma GmbH. Retardiertes Hydromorphon (Palladon ${ }^{\circledR}$ ) reduziert nicht nur signifikant die Schmerzintensität sondern verbessert zudem auch die Lebensqualität. Das zeigen zwei Beobachtungsstudien, die im Rahmen einer Poster-Prästentation beim Kongress der Deutschen Gesellschaft für Palliativmedizin (DGP) im April in Aachen vorgestellt wurden. Weitere Informationen bei

Dorothea Küsters Life Science Communication $\mathrm{GmbH}$

Dr. Georg Dönges

Tel. +49 69 61998-15, Fax -10

E-mail doenges@dkcommunications.de
MedacSchering Onkologie GmbH. Die CLL9Studie der deutschen CLL-Studiengruppe (DCLLSG) ist speziell für ältere CLL-Patienten konzipiert und schließt explizit Patienten mit altersbedingten gesundheitlichen Einschränkungen ein. In der CLL9-Studie erhalten alle Patienten ein in der Dosisintensität reduziertes Therapieregime mit Fludarabin (Fludara ${ }^{\circledR}$ ) in einer Dosierung von $30 \mathrm{mg} / \mathrm{m}^{2}$ an den Tagen 1, 3 und 5. Weitere Informationen bei

Kommunikation und Beratung im Gesundheitswesen, Melitta A. Zakel

Tel. +49 89 159-6752, Fax -70492

E-Mail zakel.pr@t-online.de

\begin{tabular}{ll}
\hline KARGER & @ 2005 S. Karger GmbH, Freiburg \\
& \\
$\begin{array}{l}\text { Fax }+497614520714 \\
\begin{array}{l}\text { E-mail Information@Karger.de } \\
\text { www.karger.com }\end{array}\end{array}$ & $\begin{array}{l}\text { Accessible online at: } \\
\text { www.karger.com/onk }\end{array}$ \\
\end{tabular}

